Artículo producto de la investigación

\title{
Algunas categorías de la cultura política y sus posibilidades como herramientas de análisis en el campo de la investigación socio-política actual en Colombia ${ }^{1}$
}

Diego Alejandro Malagón González ${ }^{2}$

Resumen

En este articulo se aborda la cuestión acerca de las posibilidades que tiene la cultura política, entendida no solamente como un objeto de estudio, sino también como un enfoque analítico propio en el desarrollo de trabajos de investigación dentro del ámbito de la ciencia política y las ciencias sociales en Colombia

Específicamente se recurre a una perspectiva micropolítica, dentro de la cual, la cultura política como subdisciplina al interior de la ciencia política aparece como instrumento interesante en la comprensión e interpretación de las actitudes de ciertos actores en el ámbito público.

Desde una perspectiva crítica se define la cultura política ${ }^{3}$ en Colombia a partir de las tensiones y contradicciones de los grupos sociales en conflicto y reconociendo la coexistencia de una pluralidad de culturas políticas en el contexto social nacional. A l interior de dicho enfoque crítico

\footnotetext{
1 Documento que hace parte de la tesis de grado presentada por el autor dentro del programa de Maestría en Administración Publica - ESAP. 2011. "La tecnocracia neoconservadora y neocorporativa en Colombia: Una comparación del discurso empresarial del Opus Dei y del Gobierno nacional: 2002-2010"

2 Politologo - Universidad Nacional de Colombia, correspondencia: rasinari@gmail.com

3 Se puede concebir la cultura política como el conjunto de prácticas y representaciones en torno al orden social establecido, a las relaciones de poder, a las modalidades de participación de los sujetos sociales, a las jerarquías que se establecen entre ellos y a las confrontaciones que tienen lugar en diferentes momentos históricos. Tales relaciones no giran solamente alrededor del Estado nacional, siendo posible identificar la existencia de diferentes culturas políticas dentro de las sociedades, al tiempo de considerar expresiones culturales no relacionadas de manera explícita con la esfera de la política pero que inciden en la constitución de modelos y pautas que repercuten sobre ella. (Herrera, Pinilla, Infante, Díaz, 2005. Pág. 34).
} 


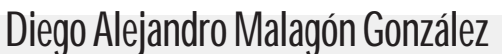

Algunas categorías de la cultura política y sus posibilidades como herramientas de análisis en el campo de la investigación socio-política actual en Colombia, artículo ...

encontraremos las diversas aristas sobre las cuales se ha estructurado la cultura política en nuestro país. Desde las perspectivas conservadoras y neoconservadoras que determinan dinámicas neodemocráticas y sus expresiones neocorporativas del régimen político colombiano en el marco del model o neol iberal de desarrollo, hasta desembocar finalmente en las tendencias y expresiones neocorporativas "patrióticas" que según algunos autores se evidencian en el país al finalizar el siglo XX y en los comienzos del siglo XXI.

Palabras ctave: Cultura política, ciencia política, micro-política, neocorporativismo

\section{Some categories in political culture and their possibilities as tools of analysis in the field of socio-political research in colombia today}

\begin{abstract}
This paper addresses the possibilities of political culture, understood not only as a topic of study, but also as an analytical approach on its own for the development of research works within the scope of political and social sciences in Colombia - specifically a micro-political approach is called into play, within which political culture emerges as a subordinated discipline within political science, as a useful tool to understand and get insights about certain actors' attitudes in the public sphere.

From a critical perspective political culture in Colombia is defined on the basis of the strains and contradictions of social groups in conflict, recognizing the coexistence of plural political cultures in the nation's social context. Inside that critical approach, the thorny questions upon which political culture has been built in our country are to be found.

From conservative and neoconservative approaches defining neodemocratic dynamics and their neo-corporate expressions in the Colombian political regime within the framework of the neo-liberal development model up to the "patriotic" neo-corporate trends and expressions that according to several authors are evidenced at the end of the 20th century and beginnings of the 21th century in our country.
\end{abstract}


Revista de

investigaciones UNAD

Volumen 09. Número 3. Diciembre 2010

Keywords: Political culture, political science, micro-politics, neocorporatism.

Recibido: 27 Marzo de 2011

Aceptado: 2 Mayo de 2011

La cultura política como enfoque analítico

Entendiendo que la cultura política como objeto de estudio y al mismo tiempo como enfogue de análisis no puede ser asumida como una categoría estatica, sino que se construye en la dinamica social como conjunto de pautas de razonamiento, de argumentacion y representacion de la realidad social antes que como conjunto cerrado de valores y creencias, surge entonces la pretension de explorar los sistemas de valores, las representaciones simbolicas y los imaginarios colectivos de los diferentes subgrupos que configuran la cultura politica colombiana, que para el caso nacjonal podria definirse de manera tajante como caracterizada por la tension innegable entre tradición y modernidad en sus dinámicas politicas.

A partir de esta caracterización y siguiendo la propuesta tipológica de B lanca M uñz en cuanto a los modelos socioculturales desde los cuales se puede abordar la cultura en general y la cultura pol itica particularmente (M uñoz, 2005), describiremos para el caso de Colombia, el modelo sociocultural conservador, el cual incl uye dentro de sí las perspectivas neoconservadoras y las neoliberales. Posteriormente en la tentativa de profundizar en la caracterizacion de la cultura pol itica de Colombia y dar cuenta de los efectos que estos modelos socioculturales tienen en la tipologia especifica de la democracia en este contexto; se desarrol lara la categoria de las denominadas neodemocracias y sus expresiones asociadas al autoritarismo.

\section{La perspectiva conservadora y el modelo neoconservador}

Dos de los principales modelos socioculturales que se encuentran insertos dentro de la perspectiva conservadora y que sirven para caracterizar la cultura política de Col ombia son: el modelo neoconservador y el modelo neoliberal. ${ }^{4}$

El modelo sociocultural neoconservador tiene como eje interpretativo el de la cul tura como jerarquia social. Sus fuentes son variadas: de una parte

4 Hay que dejar claro que el tratamiento que se hace del modelo neoliberal es el de modelo sociocultural inserto dentro de la perspectiva conservadora, sin perjuicio de su tratamiento profundo como enfoque económico o como línea de economía politica. 


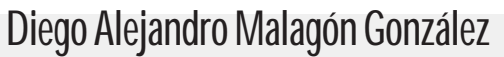

Algunas categorías de la cultura política y sus posibilidades como herramientas de análisis en el campo de la investigación socio-política actual en Colombia, artículo ...

el conservadurismo clásico (B urke, Tonnies) y su planteamiento de la desigualdad como un elemento inherente a la sociedad, la inmutabilidad de la naturaleza social, y una estructura social orgánicamente jerarquizada, legitimamente dirigida por unas minorias selectas y una defensa del contexto cultural y la preeminencia, por tanto, de la comunidad frente al individuo (M uñoz, 2005).

Pero además de su expresión filosófica, el neoconservadurismo se nutre de la teoria del fin de las ideologías (Daniel Bell, Francis Fukuyama) donde la categoria de ideol ogla es reemplazada por la de cul tura, criticando la pretenșión moderna de una sociedad justa e igualitaria y señalando como principal contradiccion cultural del capitalismo el incentivo de las politicas distributivas del Estado de bienestar, en cuanto ha generado una sobrecarga en la economía, dejando sin incentivos la iniciativa individual y la posibilidad de maximizar el mercado, siendo la secularizacion una de las causas sustanciales de horadacion del capitalismo (M uñoz, 2005: 45).

Enel marco deestospresupuestos, laconcepción cultural del neoconservadurismo defiendeel princi pio de una sociedad fundada en el liderazgo, el cuestionamiento a todas las expresiones de democracia participativa, su rechazo a los principios y derechos universal es de justicia e I qual dad sociales, asi como a toda iniciativa de cambio frente a la exaltacion de un pasado intemporal, A ello se suma una clara reivindicación de un elitismo cultural que en ultimas es politico. Siguiendo a Shumpeter, el capitalismo es la máxima expresion de progreso y por consecuencia el papel de la democracia no es otro que el de promover capitalismo, lo cual, por el grado de complejidad al canzado, solo puede ser realizado por una elite ilustrada y tecnica. En rechazo a la cultura de masas y a estil os de vida homogenizantes, el papel de la élite, ademas de manejar el sistema politico y economico, es el de restituir la jerarquia, la autoridad social y cultural horadadas (M uñoz, 2005: 48).

No es de extrañar la relación del neoconservadurismo con las nuevas derechas del capitalismo tardio, segun M uñoz, en la medida en que ha sido un catalizador de su consolidación: tradicionalismo, nacional ismos conservadores, fascismo, conservatismo ingles y americano, integrismo catolico, autoritarismos en sus diversas formas a nivel intelectual, el comunitarismo premoderno, teorías como el decisionismo amigo/enemigo de Schmitt y las nuevas interpretaciones de Lockey Hobbes seenmarcan comodamenteen estaperspectiva neoconservadora (Muñoz, 2005: 57).

La estrategia neoconservadora de democratización en Colombia

Uno de los principales postulados sobre los cuales el modelo de desarrollo neoliberal (y sus paradigmas teoricos) se ha tornado en el paradigma 
Revista de

investigaciones UNAD

Volumen 09. Número 3. Diciembre 2010

hegemónico sobre la construcción del orden social y económico (también político), ha sido sin duda, su crítica y desconfianza en el Estado como referente principal en la construccion del nuevo orden democratico. Esa critica al Estado de bienestar se sustentaba respecto a la ingobernabil idad que la expansion del mismo estaba causando en la sociedad. La ingobernabil i dad diagnosticada por los expertos neoliberal es se traducía en el desdibujamiento que a nivel social y economico se daba entre la estera publica-estatal y la esfera privada-mercado que obstacul izaba el optimo desarrollo de la sociedad y que configuraba un régimen ineficiente y corrupto. De la misma manera, la expansion incontrolada del Estado amenazaba de manera radical la libertad individual, poniendo en peligro los derechos fundamentales liberales sobre los que se sustentaba la democracia liberal.

Las demandas públicas se constituían en otra fuente de ingobernabilidad desde la perspectiva de la tecnocracia neoliberal: segun esta, el número creciente de demandas sociales y la imposibilidad de respuesta que tenia el Estado, aunado al profundo déficit fiscal, habian generado un progresivo nivel de deslegitimacion de la actividad estatal y un aumento generalizado de las protestas sociales y las expresiones armadas. Se propugna entoncess por el acomodamiento de las funciones del Estado a traves de la reduccion de las expectativas sociales con respecto a los alcances de la accion estatal, asi, el mercado se erigiria de ahora en adel ante como el encargado de satisfacer todas las expectativas de la sociedad. De este modo, el mercado lograria maximizar las libertades individuales y el Estado en últimas se constituiria en el guardian de estas ultimas;' se inauguraba entonces una nueva forma de relacionarse el Estado y la sociedad civil mediada por el fin del Estado de bienestar.

Este nuevo papel del Estado se expresaría con mayor claridad en el proyecto de acto reformatorio de la Constitución en nuestro pals:

El Estado social, entendido como idea regulativa, significaría que para el Estado existiría una obligacion de buscar la justicia social en sus actuaciones. Este debería promover la igual dad para los diferentes grupos sociales, lo cual no significa la implantáción del "iguali itarismo", sino que todos los sectores de la población deberian tener igualdad de posibilidades para el pleno goce de sus derechos. ${ }^{5}$

El nuevo Estado social de derecho promovido en la carta constitucional del 91 sería la expresión de un Estado mas eficiente y ligado de una manera mucho más productiva a la vida de los ciudadanos, los cuales, a partir de entonces, se convertirian en, los actores protagonicos en el accionar de lo público, ahora en funcion de la lógica del mercado.

5 Proyecto de acto reformatorio de la Constitución Política. 1991. Pág. 91. 


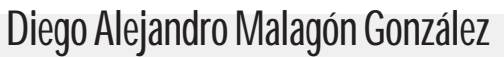

Algunas categorías de la cultura política y sus posibilidades como herramientas de análisis en el campo de la investigación socio-política actual en Colombia, artículo ...

Por su parte, el eje de la participación de esta estrategia neoconservadora de dominación se levantaba sobre la importancia de la incidencia de los individuos en los asuntos de interes colectjvo. Se abogaba entonces por una mayor participacion y un control democratico de los ciudadanos en todos los procesos sociales; asi los individuos libres se constituian en los nuevos sujetos pol iticos de este nuevo modelo de democracia. En este marco, los ciudadanos adquirian responsabilidades que anteriormente le correspondian al Estado y potenciaban libertades antes amenazadas por el monstruoso "L eviatan", lo cual en últimas, daba margenes favorables para la ampliacion de la democracia.

Es así que la idea de sociedad civil se ubicaba en el centro de la resignificación del imaginario de la democracia, construido sobre la base de la deslegitimacion del Estado y una idea de democracia centrada en la participación de la sociedad civil, entendida como el ejercicio depracticas incluyentes, deseables y posibles, que acabarian con el monopolio pol itico del liberalismo y el conservadurismo.

\section{La perspectiva conservadora y el modelo neoliberal}

Teniendo en cuenta lo anteriormente señalado, no es de extrañar entonces la íntima relación de la perspectiva conservadora con el modelo sociocultural del neoliberalismo, y. sus presupuestos de retorno a los principios económicos liberales decimononicos. Al mismo tiempo se observa la preponderancia del mercado en los ambitos económico y social, la supremacia del sector privado sobre el público, lo que implica una minimizacion del Estado, el papel que teoricos e intelectuales neol iberales, consideran positivo de la desigual dad como incentivador de la producción, la eliminacion del papel social del Estado y sus políticas redistributivas, todo resumido en la "desideologizada" deificacion del mercado.

De aquí se deriva la concepción de la cultura como mercado. Entendiendo laidea de un, mercado sin restricciones, donde la solidaridad y la simpatía son reducidas a egoísmo y calcullo de utili idad frente a los demás. Munoz destaca la forma en que Milton Friedman cuestiona el derecho privado, propende por un mercado libre sin restricciones donde la cultura queda limitada al consumo desde el reduccionismo de unas ecuaciones binarias bastante sui generis'; al ta cultura-consumo suntuario, cul tura media-consumo de masas, cul tura bruta-consumo popular (M uñoz, 2005).

A esto se suman todas las variantes del liberalismo y el individualismo metodologico sobre el orden social y cultural. El neoliberalismo basa su fundamentación epistemologica en la teoria de la eleccion racional, donde el individuo gueda emplazado a los dilemas irrenunciables de la teoria de los juegos enfrentado a decidir entre preferencias frente a opciones racionales. La cultura se reduce a opciones del consumidor frente a ofertas del mercado. 
Revista de

investigaciones UNAD

Volumen 09. Número 3. Diciembre 2010

En la misma lógica, la teoría de la elección pública (public choice) define la accion colectiva a partir del esquema estimulo-respuesta de sujetos, quedando la conducta individual y colectiva enmarcada en parametros de maximizacion de utili idades y la cultura entendida como forma de preferencia social del individuo en busqueda de maximizacion de beneficios, reduciendola a un bien de consumo donde el consumidor-receptor impone sus preferencias. La racionalidad maximizadora es la unica guia del individuo en la seleccion de sus opciones.

\section{El fenómeno de las neodemocracias}

A través de la historia del Estado moderno, el paradigma democrático se ha enfrentado al cuestionamiento constante de sus postulados debido a la incapacidad del régimen para solucionar los vacios de legitimidad, cobertura y gobernabilidad que se derivan de la intromision del sistema economico sobre el politico. Dicho aspecto se podria relacionar con la propension a la reinterpretación del sistema democrático liberal para adaptarse a las nuevas exigencias del capital y acoplar alternativas de dominacion politica que no se corresponden con los supuestos de libertad, igual dad y participacion.

De acuerdo a lo anterior, el referente democrático, se manifiesta como un referente formal que en la practica nunca pudo encontrar una realizacion plena debido a las falencias estructurales que, en la subordinacion de lo social y lo, politico al ambito economico, han desarrollado las democracias contemporaneas. Una crisis que se desencadena desde el colapso de la figura paternali ista del Estado de bienestar iniciandose la carrera de los regimenes por sobrevivir a la apatia y escepticismo politico de las masas, a la formacion de iniciativas sociales y a la perdida de legitimidad de las el ites tradicionales en el poder, En consecuencia, las democracias, en aras de conservar el orden y la estabilidad economica y social, han evolucionado en su practica y su discurso hacia sistemas hibridos de dominacion.

Este panorama presenta a las neodemocracias como sistemas que se desenvuelven en medio de la convivencia de diferentes modernidades, una simultaneidad que recoge elementos tradicionales, modernos y postmodernos; lo que evidencia al tiempo una identidad politico-cultural sustancial mente difusa que propicia e impide decantar minimamente una conciencia política definida a nivel social. Este fenomeno genera a la vez dinamicas de indiferencia y escepticismo, pol iticos, lo que se resuel ve volviendo a formas de legitimacion, para usar los terminos weberianoș, tradicional-carismáticas y, en el marco de lo anterior, a proyecciones autoritarias mimetizadas en posturas pseudoautenticas de carácter premoderno, moderno o postmoderno. 


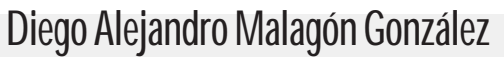

Algunas categorías de la cultura política y sus posibilidades como herramientas de análisis en el campo de la investigación socio-política actual en Colombia, artículo ...

La pérdida de influencia de la política sobre la economía, además de la despolitización del concepto de ciudadania, el desgaste de la legitimidad en la acción estatal, sumados a la supresión del espacio publico y de la participación ciudadana, desembocan entonces en el surgimiento de democracias de corte neoconservador en donde se han configurado tendencias autoritarias, neopopul istas y neocorporativistas en los sistemas democraticos contemporaneos. En sintesis, se desemboca en una tendencia a la apolitizacion social, apoyada en la individualizacion que la competencia del mercado incluye en el ciudadano, que ya no se percibe a si mismo como parte activa del espacio publico sino que se recluye en el escenario privado, donde final mente es limitado y definido por la influencia decisoria de los pequeños grupos hegemonicos que en el campo politico y economico sustentan el poder.

Estepanorama permiteuna homogenización aparente, producto de lapasividad politica, legitimando al regimen democratico, de tendencia autoritaria a determinar, un orden de valores, sustentando asi sus iniciativas economicas y de organizacion social basadas en la primacia de la seguridad, la paz y la estabilidad sobre los derechos y libertades fundamental es; la amenaza del enemigo interno y la ambiguedad y arbitrariedad en su definicion.

Por último se presentan las prácticas neocorporativistas que priorizan la participación de gremios y asociaciones en la toma de decisiones públicas de caracter económico y políicico orientadas hacia la defensa de intereses privados, una practica que socaba la legitimidad del Estado como ente administrativo y coloca a un tercer poder que no está fiscalizado, ni controlado normativamente con una posicion privilegl ada frente a otros organi smos institucionales y sobre todo frente al conglomerado social como tal.

\section{El neocorporativismo}

El neocorporativismo es un producto del enfrentamiento de las democracias, con las consecuencias que implica, en todos los planos, el desarrollo del sistema economico y político neoliberal. El corporativismo se presenta, en palabras de Claus Offe, como "esquemas potenciales de regulacion que en ningun caso estan previstos en el Estado democratico constitucional; su existencia se contempla a nivel fáctico y no consideran un entramado normativo que las regule desde la institucionalidad" (Offe, 1996; 135).

Estos esquemas se expresan en la asociación y agrupación de intereses, interactuando con el gobierno, la administracion, las instituciones publicas participativas, asi como las instituciones de, competencia entre los partidos. sin embargo, a pesar de su interaccion, no están contenidas en ninguna de estas 
Revista de

investigaciones UNAD

Volumen 09. Número 3. Diciembre 2010

formas; su presencia se justifica y de cierta forma se ratifica a nivel funcional más allá del nivel formal y de control eficaz del entramado legal democratico. Su funcion de representacion de sectores sociales se compagina con el de la ordenación e influencia sobre diferentes campos como la educación, la politica economica, el bienestar social, el minimo salarial, etc.

Como ya lo avizoraba Touraine, el desarrollo de este tipo de organizaciones responde, en primer termino, a un vacio parlamentario e institucional, que permite la apertura de espacios de incidencia directa sobre la politica pública. En tal sentido, la política pactista y de negociacion extrainstitucional, que supone un sistema neocorporativista paralel o a un Estado de derecho, supone la preocupacion de que se estén transfiriendo, del parlamento, los partidos y el Estado, atribuciones y competencias en áreas politicas importantes a estructuras que, como apunta Offe, cobran el estatus de gobiernos privados (Offe, 1996: 134). Es asi que se percibe la cesión de poder pol itico a un sector focalizado, ciertamente reducido de la poblacion, siendo esta una situacion que no presenta control constitucional alguno, por lo que resulta necesaria una revision de los supuestos neocorporativistas, para comprender cual es su papel en las democracias contemporaneas.

La tensión entre mercado, regulacción estatal y autocompromiso comunitario define la relacion entre la actuacion individual y la racional idad, colectiva del sistema. La intervención estatal se agota como modelo después del fracaso del model o de bienestar y la crisis de eficacia-eficiencia que ello representa; la intervencion del mercado resulta violenta y excluyente, demostrando no ser la clave para satisfacer las expectativas de todos los que participan en el sistema economico neoliberal; resul ta, por ende, el neocorporativismo como la busqueda de espacios que han sido vetados.

En los años setenta, junto a la crisis del Estado nacional y de los partidos políticos, y su reemplazo por el neoliberal, reaparece el corporativismo en una versión denominada corporativismo "organizado" (Schmilter, 1998), Un corporativismo ostensiblemente político que nace con la inscripción orgánica de los agentes gremiales en el complejo que atiende los asuntos comunes y a través de los cuales se adoptan las normas de orden general. Los aparatos centrales de Estado, el gobierno, las asambleas legislatjvas y los mandos de la burocracia, en fin, el sistema superior de decision politica, que se compone con representaciones funcionales, titulo de complemento o bien de recorte, de las intervenciones ciudadanas (Schmitter, 1998: 20-21).

Estas prácticas despliegan un nuevo modo de hacer política, cambiando la forma de producción de los poderes y de el aboracion de consensos, tejiendo un șistema particular de compromisos que reclama una dossis importante de gobierno por negociacion - un proceso de contractualizacion marcado-. En 


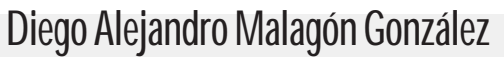

Algunas categorías de la cultura política y sus posibilidades como herramientas de análisis en el campo de la investigación

socio-política actual en Colombia, artículo ...

fin, un sistema, que establece un régimen de compensaciones internas para amortiguar los efectos de la liberalizacion y de la apertura, a su vez sirviendo como elemento de alianza y de compromisos de intereses.

El nuevo corporativismo opera, entonces, mediante la política de concertación subordinada que fortalece el papel rector y el dominio de los sectores comprometidos con la política neoliberal, dando paso a un pluralismo acotado que abarca a la sociedad, los partidos politicos opositores y a la propia estructura de los partidos oficiales, sobre la base de reconocer "organismos representativos" que les otorgan el monopolio de la representación en sus ámbitos de acción respectivos, a cambio de que respeten ciertos controles sobre la selección de sus dirigentes y articulen sus demandas y apoyos a los programas gubernamentales.

Los organismos empresariales son incorporados a comisiones y consejos en los que se decide a quien beneficia la politica economica, quien es excluido del mercado a traves de las normas de calidad o los requisitos para participar en licitaciones; o quien está en condiciones para recibir los beneficios de los programas de apoyo a la industria. De esta manera, es el propio gobierno el que decide quienes son los "interlocutores validos" y "verdaderamente representativos" del sector empresarial (Salcedo, 2006).

El sustento del corporativismo se basa en dos premisas, la primera, un compromiso con la corporación, de tal forma que las disposiciones y decisiones de esta sean las propias, despojandose de todo afan individual. Citando a Hegel, de ello resul ta una "actividad consciente para un objetivo comun". Por otro lado está la necesidad de la existencia de grupos intermedios entre el Estado y la sociedad, grupos que esten lo suficientemente cerca del ciudadano como para incluirlo en su radio de accion; en ello reside su pertinencia como sujeto social. Sin embargo, estos supuestos de solidaridad y responsabilidad en la representacion que soportan su existencia en la ocupacion indirecta de "mis" problemas e intereses mediante la ocupacion directa en los problemas del sistema, se enfrentan con diferentes controversias. En primer término, los miembros no tienen un efecto decisivo a nivel individual; la prerrogativa de la decision esta infl uenciada por la necesidad de la corporacion de comprometer a sus miembros para, con criterio unificado, proceder a una negociacion exitosa. Este primer recorte de las libertades se acompana de la influencia y la atribucion que estos grupos se adjudican para participar en procesos de decision sobre desarrollo socioeconomico y regulación publica. Esto realza la exclusion de una gran mayoria frente a un proceso decisorio de gran impacto en sus vidas. En tal sentido, offe argumenta que cuanto más se compl ete y reccorte el principio de representacion territorial con el de la representacion funcional, más afectada ha de verse la norma de igualdad ciudadana por el hecho de la desigualdad economica de los ciudadanos (Offe, 1996: 153). 
Revista de

investigaciones UNAD

Volumen 09. Número 3. Diciembre 2010

La exclusión del no propietario se evidencia en el aval de movimientos de organizacion de propietarios por una organización corporativa políticamente influyente. Al recurrir a estos mecanișmos de regulacion poli itica semioficiales, se está sugiriendo una reinterpretacion que debera ser leida en un contexto más amplio, que examine de lleno el impacto que esto genera en ciudadanos excjuidos politica y economicamente de un proceso publico de decision politica y de refuerzo o respaldo a las reacciones apáticas o las iniciatjvas movimientistas que se presentan en las neodemocracias. Se retrocede asl al concepto precario de democracia restringida.

Según Schmitter, el corporativismo opera a nivel "meso", "micro" e incluso "macro", mediante intercambios bilaterales entre empresarios y sindicatos de diferente rango con la intervencion del gobierno y los partidos, en convocatorias centrales y mas frecuentemente descentral izadas produciendo convenios sectoriales y algunas instancias de dialogo general en la forja de un corporativismo diversificado, de sello "comperitivo" y plural, que se compagina con la reubicacion del Estado y el "individualismo de mercado" (Schmitter, 1998: 6). El corporativismo, que se define como "una doctrina que propugna la organizacion sobre la base de asociaciones representativas de los intereses y de las actividades profesionales - corporaciones-, mas que por individuos, se propone por via de la solidaridad organica de sus, intereses concretos y de colaboracion derivada, la remocion o neutralización de los elementos conflictivos, es decir, de la competencia economica, la lucha de clases sociales y de la diferenciación ideologica a traves de un poder politico que debe conciliar esos intereses sectoriales con cierto "supremo" interés patriotico" (Schmilter, 1998: 8).

Por medio del orden corporativista se otorga al trabajo el estatus global y la funcion de un principio ordenado en la sociedad y la economia. Es asi que, a la manera de un sistema politico, el corporativismo se relaciona directamente con la problematica de como el Estado puede sostener un orden social y económico dentro de un orden donde predomina la producción del sector privado. En tal contexto, el Estado empresario tiene la funcion principal de establecer y mantener un orden económico y social, y las instituciones liberales democraticas pueden no existir en absoluto, siendo ahora las organizaciones de productores - y no los partidos- las que intentan jugar el rol de interlocutoras del Estado, desempenando no sólo la funcion de representar sino de operar como agencias reguladoras a favor del Estado.

El corporativismo ya había renacido en loș inicios del proceso moderno de la industrial izacion como protesta contra la mișma empresa capitali sta, su sistema y su revol ución pol ítica, al tenor del pensamiento catól ico que afirmaba que los principios modernos de la igual dad e individualismo apl astaban al individuo exponiendolo a la fuerza dominante del trabajo y la sociedad politica. El ideal restaurativo de una sociedad inmovil pero organica y jerárquica, sin conflictos 


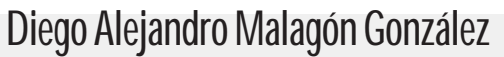

Algunas categorías de la cultura política y sus posibilidades como herramientas de análisis en el campo de la investigación socio-política actual en Colombia, artículo ...

ni antagonismos, éticamente lograda ${ }^{6}$ Io proponen los dirigentes legitimistas catolicos a traves de articulaciones sol idaristas que empalmaran a los excluidos del sistema (Bobbio y Matteucci, 1982).

Reaparición de las tendencias neocorporativas y el discurso corporativo católico Esta dinámica corporativa reaparece con fuerza hacia los años ochenta, ahora con el anadido de la nocion de patriotico en el pensamiento y accion de los militantes católicos y de los partidarios populistas conservadores y monarquistas espanoles, quienes encuentran su fuente en el pensamiento social de la iglesia, actualizado en la vida y obra del papa J uan Pablo II.

En el documento titulado "Nacionalismo, Patriotismo y Subsidiaridad, el IV Congreso Católicos y Vida Pública, Desafíos globales:' La doctrina Social de la I glesia hoy" (Castro. Velarde, 2003), se señala que "el cuarto mandamiento de la ley de D ios establece, entre otras, las obligaciones que tenemos con nuestra patria, que muchas veces son olvidadas". Citando tambien al Papa Juan Pablo II en su discurso a los obispos de A rgentina en 1982, se declara que el patriotismo integra la universali idad; "Son los padres y la patria los principios secundarios a quienes, después de Dios, los hombres les son deudores. De modo que después de la virtud de la religion, cuyo papel es rendirle un cul to a Dios, viene la virtud de piedad, que rinde un culto a los padres y a la patria" (Castro. Velarde, 2003: 652)

Esta perspectiva pretende demostrar con su reflexión la superación de "la dialéctica del conflicto que para Hegel y Marx es el motor de la historia", para insinuar el solidarismo de las clases y la inexistencia de las izquierdas y derechas, señalando que "los procesos de integración han de permannecer siempre abiertos, estando al final de este proceso como vinculo de unión de todos los hombres, la unidad superior y última, de toda la catolicidad", Esto se opone a la teoria revolucionaria de la soberanía nacional y al principio de las nacionalidades que habian inspirado las revoluciones liberales burguesas de mediados del siglo XIX.

Consecuente con laglobalización y su neoconservadurismo, la descentral ización y el solidarismo, la doctrina concibe el principio de subsidiaridad, "segunda descentralizacion" y el patriotismo, fundamentado en la enciclica Centesimus A nnus N ${ }^{\circ} 48$ del Pontifice, según la cual las estructuras sociales de orden superior" "no deben interferir en la vida interna de un grupo de orden inferior, privándole de sus competencias, sino que más bien debe sostenerle en caso de necesidad y ayudarle a coordinar su accion con miras al bien comun".

La subsidiaridad abarca el respeto de la autonomía de la persona sobre el colectivo, es contrario al nacionalismo, "porque pretende crear una nación

6 En 1892, en la Encíclica Rerum Novarum, el modelo corporativo asume una configuración doctrinaria más precisa al afirmar que a "la solución de la cuestión obrera pueden contribuir mucho los capitalistas y los obreros mismos, con instituciones ordenadas para ofrecer oportunos auxilios a las necesidades y para reconciliar y unir a las dos clases entre sí". (V. Bobbio, Norberto y Matteucci. 1982. Diccionario de Política D-J. Siglo XXI editores. Bogotá, Colombia. Pags. 431- 438). 
Revista de

investigaciones UNAD

Volumen 09. Número 3. Diciembre 2010

uniforme acabando con los derechos de las entidades intermedias, cuerpos sociales, regiones y minorías", al pretender acabar "con una unidad superior identificando nacion con Estado con base en el principio de las nacionalidades revolucionarias que olvida que por debajo de ella misma estan las "comarcas, municipios, corporaciones y familias".

La subsidiaridad comienza por la familia, célula básica de la sociedad, y "la segunda descentralizacion" se realiza hacia los municipios donde el patriotismo, "al ser un concepto que se incardina en la virtud de la piedad y que va desde lo más cercano hasta lo más lejano, puede indudablemente servir de base para llevar a la practica el principio de subsidiaridad, ya que como nos ha recordado |uan Pablo II, el amor a la patria no excluye sino al contrario fomenta la necesaria solidaridad mundial" (Castro. Velarde, 2003: 658).

\section{Conclusiones}

Los estudios sociológicos y sociopolíticos en los últimos años se han enfocado en examinar los procesos de configuracion de la accion de los diversos grupos sociales, mas aun cuando ese accionar tiene implicaciones en la configuracion de los procesos sociopolíticos en términos de la infl uencia o crítica que determinada accion colectiva tenga frente al funcionamiento de los sistemas sociales.

En este sentido, la cultura política como subdisciplina inserta dentro de la ciencia politica, que a la vez que aparece como objeto de estudio y enfoque analitico de los fenomenos sociopolíticos, nos ofrece una serie de categorias analíticas que resultan interesantes al momento de examinar las dinamicas específicas en nuestro contexto sociopol ítico por parte de sus diversos actores.

Las categorías pertenecientes a la cultura política que se han propuesto en este escrito se tornan en este sentido funcionales y potencial menteoperacionalizables al momento de tratar de explicar los fenomenos politicos que se expresan al comenzar y transcurrir el siglo XXI en America Latina y especificamente en nuestro pals. Los nuevos procesos de insercion en la globalizacion y los modelos de desarrollo de nuestros paises que se aj ustan a estos procesos, van definiendo y configurando las diferentes estrategias, cosmovisiones, idearios e ideologias de los distintos actores y sectores con poder.

Estas categorías pues, nos darían una primera oportunidad de análisis desde una perspectiva critica de la cultura política, del diverso accionar de los sectores con poder, lo que a su vez nos permitirá entender y comprender. los mecanismos por los cual es estos sectores pretender influir (o, lo hacen efectivamente) en los procesos de toma de decisiones a nivel de lo publico en estos contextos. 


\section{DegoAlejandoMkagónconzdez}

Algunas categorías de la cultura política y sus posibilidades como herramientas de análisis en el campo de la investigación socio-política actual en Colombia, artículo ...

\section{Referencias bibliográficas}

Bobbio, Norberto y Nicola Matteucci. 1982. Diccionario de Política D-J.Bogota. Siglo XXI editores.

Castro Velarde, J osé. S.F. IV Congreso Católicos y Vida Pública. “Desafíos globales: la Doctrina Social de la Iglesia hoy. Vol. 2, 2003, , págs. 647-664

Herrera, Martha Y Pinilla, Alexis, 2005, La construcción de La cultura política en Colombia, Bogotá. Universidad Pedagógica Nacional.

Muñoz, Blanca, 2005, Modelos Culturales. Teoría Sociopolítica de la Cultura. Barcelona. Anthropos.

Offe Claus. 1996. "Presupuestos del neocorporativismo", en Partidos Políticos y nuevos movimientos sociales. Madrid. Alianza Editorial. Págs. 128-162.

Salcedo García, Pedro. «Corporativismo. Democracia y Sociedad civil». Mayo 8 de 2006. En http://www.diputados.gob.mx/cronica57/contenido/cont12/anali6.html. Fecha de consulta: 16/08/2011

Schmitter, Philippe, et al. 1998. El fin del siglo del corporativismo. Caracas. Editorial Nueva Sociedad. 